TITLE:

\title{
Neural correlates of perceptual grouping effects in the processing of sound omission by musicians and nonmusicians.
}

\section{$\operatorname{AUTHOR}(\mathrm{S})$ :}

Ono, Kentaro; Altmann, Christian F; Matsuhashi, Masao; Mima, Tatsuya; Fukuyama, Hidenao

\section{CITATION:}

Ono, Kentaro ... [et al]. Neural correlates of perceptual grouping effects in the processing of sound omission by musicians and nonmusicians.. Hearing research 2014, 319: 25-31

\section{ISSUE DATE:}

2014-11-07

URL:

http://hdl.handle.net/2433/192475

\section{RIGHT:}

(c) 2014 Elsevier B.V.; この論文は出版社版でありません。引用の際には 出版社版をご確認ご利用ください。; This is not the published version. Please cite only the published version. 


\author{
Elsevier Editorial System(tm) for Hearing Research \\ Manuscript Draft
}

Manuscript Number: HEARES-D-14-00093R3

Title: Neural correlates of perceptual grouping effects in the processing of sound omission by musicians and nonmusicians

Article Type: Research paper

Keywords: Perceptual grouping; Omission; Predictive coding theory; Magnetoencephalography; Musical experience.

Corresponding Author: Dr. Kentaro Ono,

Corresponding Author's Institution: Kyoto University

First Author: Kentaro Ono

Order of Authors: Kentaro Ono; Christian F Altmann; Masao Matsuhashi; Tatsuya Mima; Hidenao Fukuyama

Abstract: Perceptual grouping is the process of organizing sounds into perceptually meaningful elements. Psychological studies have found that tones presented as a regular frequency or temporal pattern are grouped according to gestalt principles, such as similarity, proximity, and good continuity. Predictive coding theory suggests that this process helps create an internal model for the prediction of sounds in a tone sequence and that an omission-related brain response reflects the violation of this prediction. However, it remains unclear which brain areas are related to this process, especially in paying attention to the stimuli. To clarify this uncertainty, the present study investigated the neural correlates of perceptual grouping effects. Using magnetoencephalography (MEG), we recorded the evoked response fields (ERFs) of amateur musicians and nonmusicians to sound omissions in tone sequences with a regular or random pattern of three different frequencies during an omission detection task. Omissions in the regular sequences were detected faster and evoked greater activity in the left Heschl's gyrus (HG), right postcentral gyrus, and bilateral superior temporal gyrus (STG) than did omissions in the irregular sequences. Additionally, an interaction between musical experience and regularity was found in the left HG/STG. Tone-evoked responses did not show this difference, indicating that the expertise effect did not reflect the superior tone processing acquired by amateur musicians due to musical training. These results suggest that perceptual grouping based on repetition of a pattern of frequencies affects the processing of omissions in tone sequences and induces more activation of the bilateral auditory cortex by violating internal models. The interaction in the left HG/STG may suggest different styles of processing for musicians and nonmusicians, although this difference was not reflected at the behavioral level. 


\section{Highlights:}

- Sound omission in a tone sequence elicits the omission-related response (OR).

- We studied the effect of perceptual grouping on ORs.

- ORs were measured in musicians and nonmusicians using magnetoencephalography.

- ORs were increased by perceptual grouping and localized in the auditory cortex.

- The grouping effect was left-side dominant in musicians but not in nonmusicians. 
1 Proposed journal section: Research Papers

2

3

4

$5 \quad$ Neural correlates of perceptual grouping effects in

6 the processing of sound omission by musicians and nonmusicians

10 Kentaro Ono, Christian F. Altmann, Masao Matsuhashi, Tatsuya Mima, and

Human Brain Research Center, Graduate School of Medicine, 
20 Address correspondence to:

21 Kentaro Ono, $\mathrm{PhD}$

22 Human Brain Research Center, Graduate School of Medicine

23 Kyoto University, Kyoto, 606-8507, Japan

24 Phone: $++81-75-751-3695$

25 Fax: $++81-75-751-3202$

26 E mail: onoken@kuhp.kyoto-u.ac.jp

27

28

29 Keywords:

30 Perceptual grouping, Omission, Predictive coding theory, Magnetoencephalography,

$31 \quad$ Musical experience

32

33 Abbreviations:

34 OR, omission-related response; MEG, magnetoencephalography; HG, Heschl's gyrus;

35 STG, superior temporal gyrus; MMN, mismatch negativity; ISI, inter-stimulus interval;

36 AC, auditory cortex; MRI, magnetic resonance imaging; RMS, root mean square;

37 ANOVA, analysis of variance; RT, reaction time; SD, standard deviation. 


\section{Abstract}

Perceptual grouping is the process of organizing sounds into perceptually meaningful or temporal pattern are grouped according to gestalt principles, such as similarity, proximity, and good continuity. Predictive coding theory suggests that this process helps create an internal model for the prediction of sounds in a tone sequence and that an omission-related brain response reflects the violation of this prediction. However, it remains unclear which brain areas are related to this process, especially in paying attention to the stimuli. To clarify this uncertainty, the present study investigated the neural correlates of perceptual grouping effects. Using magnetoencephalography (MEG), we recorded the evoked response fields (ERFs) of amateur musicians and nonmusicians to sound omissions in tone sequences with a regular or random pattern of three different frequencies during an omission detection task. Omissions in the regular sequences were detected faster and evoked greater activity in the left Heschl's gyrus (HG), right postcentral gyrus, and bilateral superior temporal gyrus (STG) than did omissions in the

56 irregular sequences. Additionally, an interaction between musical experience and regularity was found in the left HG/STG. Tone-evoked responses did not show this 58 difference, indicating that the expertise effect did not reflect the superior tone 
59 processing acquired by amateur musicians due to musical training. These results suggest

60 that perceptual grouping based on repetition of a pattern of frequencies affects the

61 processing of omissions in tone sequences and induces more activation of the bilateral

62 auditory cortex by violating internal models. The interaction in the left HG/STG may

63 suggest different styles of processing for musicians and nonmusicians, although this

64 difference was not reflected at the behavioral level.

65

66

67

68

69

70

71

72

73

74

75 


\section{Introduction}

78 In an orchestral performance, a musical piece is produced by multiple sequences of

79 tones played in parallel. The auditory system can extract the structural components of

80 the piece, such as its melody and rhythm, from this mixture of tones using processes

81 that integrate acoustic information over time. Together, these processes are called

82 perceptual grouping, and psychological studies have identified the rules for grouping

83 sound features, such as similarity, proximity or good continuity (Bregman, 1990;

84 Deutsch, 2012; Koffka, 1935). Bregman (1990) suggested that two types of perceptual

85 grouping exist: one is stimulus-driven and works preattentively in a short time window,

86 while the other requires higher cognitive functions such as attention and/or

87 experience-based knowledge, and has a longer time window.

A wording to predictive coding theory, cortical circuits create internal models

to generate predictions about incoming stimuli (Friston and Kiebel, 2009a, 2009b;

90 Friston, 2005). An evoked response may occur reflecting the transient expression of a

91 prediction error, which results from comparison between the bottom-up inputs from

92 lower cortical/subcortical areas and top-down predictions from higher cortical areas.

93 Several studies have applied this theory to explain the early stages of auditory

94 processing (Bendixen et al., 2012; Winkler and Czigler, 2012; Winkler, 2007; Winkler et 
95 al., 2009). Key to this explanation is that an internal model is created by spectral or

96 temporal regularity, which is extracted from a tone sequence. Mismatch negativity

97 (MMN) and the omission-related response (OR), both of which are elicited by deviation

98 (a deviant tone or the omission of a tone) from a sequence of repetitive tone stimuli, can

99 be interpreted as resulting from the violation of the prediction. In particular, the OR is

100 suitable for investigating prediction-related brain activity because it does not overlap

101 with the response elicited by the stimulus. Previous studies have shown that an OR can

102 be elicited by a tone omission in an unattended tone sequence at an inter-stimulus

103 interval (ISI) of less than $200 \mathrm{~ms}$ (Alain et al., 1989; Hughes et al., 2001; Raij et al.,

104 1997; Snyder and Large, 2005; Tarkka and Stokic, 1998; Todorovic et al., 2011;

105 Wacongne et al., 2011; Yabe et al., 2001, 1997). Together with Bregman's idea and the

106 predictive coding theory, these results can be interpreted as resulting from a violation of

107 a prediction based on pre-attentive perceptual grouping based on temporal regularity.

108 The OR in the absence of attention is localized in the auditory cortex (AC) (Raij et al.,

109 1997; Todorovic et al., 2011), which may be involved in prediction and pre-attentive

110 perceptual grouping. 
113 Joutsiniemi and Hari, 1989; Penney, 2004). These results suggest that an OR to tone

114 sequences with long ISI can occur as a result of a violation of a prediction based on

115 attentive perceptual grouping. However, the neural correlates of this phenomenon

116 remain unclear. Thus, we aimed to find the neural correlates of prediction based on

117 attentive perceptual grouping in a tone sequence with a regular frequency pattern. We

118 hypothesized that, when participants paid attention to the stimuli, a repetitive frequency

119 pattern would cause perceptual grouping and help create stronger predictions about

120 incoming stimuli, compared to a tone sequence with a random pitch pattern. Thus, a

121 violation of this prediction by an omission in a tone sequence with a pitch pattern would

122 evoke a stronger OR than would an omission in a random tone sequence. To clarify this

123 issue, we compared the brain magnetic responses evoked by omissions in regular and

124 random tone sequences using magnetoencephalography (MEG). In addition, we evaluated the impact of musical experience on the grouping effect. Musical training normally includes the structural analysis of musical pieces, which should improve the ability to extract regular patterns from a tone sequence because the structural components of a piece (e.g., melody, chord progression, meter, etc.) are established by pitch and/or rhythm patterns. Although psychological studies have demonstrated that perceptual grouping depends on experience (Bhatara et al., 
131 2013; Dewar et al., 1977; Gobet and Simon, 1996; Idson and Massaro, 1976; Iversen et

132 al., 2008; Saariluoma, 1989; Simon and Chase, 1973), no study has investigated the

133 neural correlates of this phenomenon. Thus, we tested the hypothesis that musical

134 training influences the brain mechanisms involved in the perceptual grouping of

135 frequency patterns, leading to more pronounced patterns of cortical activation in 136 musicians than in nonmusicians.

137

138

\section{Methods}

140

\subsection{Participants}

141 The participants consisted of 13 amateur musicians (7 males and 6 females) who

142 regularly played musical instruments, such as piano, guitar, violin, and cello, with an

143 average experience of $13 \pm 5$ years (mean \pm standard deviation [SD]), and 14

144 nonmusicians (11 males and 3 females) who had no instrumental experience, except for

145 lessons in school. All participants were right-handed with an average age of $22 \pm 2$ years

146 and provided written informed consent to participate in the experiment. Although we

147 did not measure the participants' hearing thresholds, none of them reported difficulty in

148 discriminating the stimuli. The participants also did not report any neurological or 
149 hearing problems. The experiment was performed in accordance with the ethical

150 standards of the Declaration of Helsinki and the guidelines approved by the local ethics

151 committee of the Graduate School of Medicine and Faculty of Medicine, Kyoto

152 University.

153

$154 \quad 2.2$ Stimuli

155 Pure tones (50-ms duration, 5-ms onset/offset ramps, $65 \mathrm{~dB}$ SPL) with three different

156 frequencies (C5: $523 \mathrm{~Hz}, \mathrm{E} 5: 659 \mathrm{~Hz}$, and G5: $784 \mathrm{~Hz}$ ) were created as wave files using

157 the Audacity software program (ver. 2.0.3; http://audacity.sourceforge.net/). A silent

158 period with a length of $500 \mathrm{~ms}$ was created as the omission stimulus. Each tone

159 sequence was constructed of these tones, presented either in a regular pattern of "CEG"

160 (regular sequence) or pseudo-randomly (irregular sequence), with an ISI of $450 \mathrm{~ms}$ (Fig.

161 1A). In the irregular sequence, randomization was controlled so as not to present the same frequency more than three times consecutively, and at least three tones were presented between omissions.

166 Participants were seated in a chair in a magnetically shielded room. The tone sequences 
were presented through earphones, which was coupled to the ear by a silicon tube and was connected to an amplifier (Roland SRQ-2031, Roland Corporation, Hamamatsu, Japan) outside of the shielded room. Participants were instructed to press a button with

171 their right index finger as quickly as possible upon noticing any omission in the sequence. Because perceptual grouping facilitates the processing of deviant stimuli in a tone sequence (Idson and Massaro, 1976; Jones et al., 1982; Mondor and Terrio, 1998; Royer and Garner, 1970), the response time was used to characterize the effect of perceptual grouping.

Each sequence was presented in three separate blocks. Six blocks were conducted in total, and the order of the blocks was randomized between participants. In each block, approximately $7 \%$ of the tones were replaced with a silent period. In total, 2520 tones and 180 omissions (60 omissions for each tone) were presented in regular and irregular sequences. An additional restriction for the regular sequence was that, after each omission, the sequence started again from the $\mathrm{C}$ tone (e.g., CEGCEGCE_CEG...) to maintain the repetition of the CEG pattern. 
had.

186

187

\subsection{MEG acquisition}

188 Event-related fields (ERFs) were recorded with a 306-channel whole-head

189 magnetoencephalography (MEG) system (Vectorview, Elekta Neuromag Oy, Finland).

190 The head position was determined using four indicator coils attached to the scalp. In

191 addition, three head landmarks (the nasion and bilateral preauricular points) and head

192 shape were recorded for each participant using a spatial digitizer (Polhemus Inc.,

193 Colchester, VT, USA) before the experiment. These data were used for co-registration

194 with the $\mathrm{T} 1$ anatomical image of each participant obtained using a $0.2 \mathrm{~T}$ magnetic

195 resonance imaging (MRI) machine (Signa Profile, GE Health Care, Waukesha, WS,

196 USA). The ERFs were recorded with a band-pass filter (0.1 to $200.0 \mathrm{~Hz}$ ) and a sampling

197 rate of $600 \mathrm{~Hz}$. To reduce external noise, we used spatiotemporal signal space separation

198 (tSSS) methods (MaxFilter, Elekta Neuromag Oy, Helsinki, Finland) with a correlation

199 window of $900 \mathrm{~s}$, which covered the entire length of each block, and a correlation limit

200 of 0.980 . The acquired data were low-pass filtered using a fifth-order Butterworth

201 zero-phase filter with a cut-off frequency of $40 \mathrm{~Hz}$. The time window of each epoch 
was used for baseline correction. Peak-to-peak differences of more than $3.0 \mathrm{pT} / \mathrm{cm}$ were used as rejection criteria.

207 To analyze the temporal waveform of the brain response evoked by omission at the

208 sensor level, we calculated the root mean square (RMS) values of 20 planar

209 gradiometers that separately covered the temporal lobe in the right and left hemispheres.

210 The same array of sensors was employed in all participants. Because the observed

211 waveforms of the brain response evoked by omission exhibited a gradual increase with

212 no specific peak, the mean value from 100 to $400 \mathrm{~ms}$ after omission onset was analyzed

213 using four-way analysis of variance (ANOVA) with the factors musical experience,

214 regularity, position of omission ( $\mathrm{C}, \mathrm{E}$, or $\mathrm{G}$ tone), and laterality (left or right

215 hemisphere) using R software (ver. 2.15.2; R Foundation for Statistical Computing,

216 Vienna, Austria; http://www.R-project.org). Post-hoc analyses were conducted using

217 lower-level ANOVAs and paired $t$-tests with multiple comparison using Shaffer's

218 modified Bonferroni correction (Shaffer, 1986). 
221 To localize the possible source of the OR and compare the activation of the source

222 between conditions, we used an empirical Bayesian approach, as implemented in SPM8

223 (Welcome Trust Centre for Neuroimaging, University College London, UK).

224 Participants' T1 images were normalized to the Montreal Neurological Institute (MNI)

225 brain template, and the inverses of the parameters were used to wrap a cortical template

226 mesh to each individual MR space. Co-registration between the MEG sensor positions

227 and T1 images was achieved by manually detecting three fiducial points in both the MR

228 image and the head-shape measurement taken using the spatial digitizer. To generate the

229 forward model, the lead-field for each sensor was calculated for the dipoles at each

230 point in the cortical mesh using a single shell model. The model was then inverted using

231 the multiple sparse priors (MSP) algorithm (Friston et al., 2008; Mattout et al., 2006).

232 To evaluate the cortical distribution evoked by omission in detail, reconstructed maps

233 were created for each 100-ms time window from 100 to $400 \mathrm{~ms}$ after omission onset.

234 These maps were exported as three-dimensional images into the MNI space and smoothed using a Gaussian filter with a 12-mm full-width at half maximum (FWHM).

For group analysis, general-linear-model-based statistical analysis with random 
239 tones between 100 and $400 \mathrm{~ms}$ after omission onset were pooled separately for the

240 regular and irregular sequences and analyzed by one-sample $t$-tests, comparing the

241 activation with zero, at an uncorrected threshold of $p<0.005$. To investigate the effects

242 of the experimental variables, we conducted a three-way ANOVA with the factors

243 musical experience, regularity, and position of omission at an uncorrected threshold of $p$

$244<0.001$ for each $100 \mathrm{~ms}$ window from 100 to $400 \mathrm{~ms}$ after omission onset. All maps

245 were projected to the MNI template. The MNI coordinates of these voxels were then

246 converted to Talairach space using GingerALE (Laird et al., 2010), and Talairach Client

247 was used for anatomical labeling (Lancaster et al., 2007). To further investigate the time

248 course of the contribution of activated areas, we conducted region of interest (ROI)

249 analysis. The amplitude of each dipole in a 10-mm diameter circle centered upon the

250 selected ROI in the cortical mesh was averaged for each time point for each participant.

251 The mean of these values at each 100-ms time window from 100 to $400 \mathrm{~ms}$ was then

calculated. The ROI activity was then analyzed using ANOVAs.

To test the possibility that the effect of musical experience on the OR simply

reflected the larger brain response elicited by tones in musicians, as shown in previous 
analyzed using a three-way ANOVA with the factors musical experience, regularity, and position of omission.

259

260

\subsection{Behavioral data analysis}

261 The time difference between the onset of omission (the time at which the missing tone had been expected) and the button press was calculated as reaction time (RT). The mean and SD of the RT were analyzed using a three-way ANOVA with the factors musical experience, regularity, and position of omission. Post-hoc analyses were conducted using lower-level ANOVAs and paired $t$-tests with multiple comparisons using Shaffer's modified Bonferroni correction (Shaffer, 1986).

\section{Results}

\subsection{Behavioral data}

271 The group mean RT is presented in Fig. 1B. A three-way ANOVA with the factors musical experience, regularity, and position of omission showed main effects of regularity $(F[1,25]=5.24, p=0.031)$ and position of omission $(F[2,50]=6.68, p=$

274 0.002), although neither a main effect nor an interaction related to musical experience 
275 were observed. Multiple comparisons revealed that the omission of the $\mathrm{C}$ tone was

276 detected faster than omissions of the other tones. The three-way ANOVA of the SD also

277 showed main effects of regularity $(F[1,25]=7.71, p=0.010)$ and position of omission

$278(F[2,50]=6.54, p=0.003)$. Multiple comparisons revealed that the SD for the

279 omission of the $\mathrm{C}$ tone was larger than omissions of the other tones. The rate of correct

280 detection for the omissions was over 95\%, and did not vary significantly across

281 conditions.

282

283

(Fig. 1 around here)

\subsection{Analysis of magnetic fields evoked by omissions}

286 An example of the ERF waveform for one musician (Fig. 1C) is typical in not showing

a clear peak; instead, the amplitude increased gradually after $100 \mathrm{~ms}$ of omission onset.

The group means of the RMS values of the responses evoked by the omissions are

plotted in Fig. 2. A four-way ANOVA with the factors musical experience, regularity,

position of omission, and laterality showed main effects of regularity $(F[1,25]=30.04$,

$p<0.001)$ and laterality $(F[1,25]=6.27, p=0.019)$, although no main effect or 
293 omission evoked a larger brain response for regular than for irregular sequences,

294 irrespective of musical experience (Fig. 3).

295

296

297

298

299

300

301

302

303

304

305

306

307

308

309

310
(Fig. 2 and Fig. 3 around here)

Fig. 4A depicts the average cortical activation between 100 and $400 \mathrm{~ms}$ after

omission onset. Despite the lack of stimulus input, activation was observed in the

bilateral temporal and frontal lobes. Three-way ANOVAs with the factors musical

experience, regularity, and position of omission for each $100 \mathrm{~ms}$ time window from 100

to $400 \mathrm{~ms}$ after omission onset showed similar results. From 100 to $200 \mathrm{~ms}$, the analysis

showed a main effect of regularity in the left Heschl's gyrus (HG) and right postcentral

gyrus, as well as an interaction between musical experience and regularity in the left

HG. From 200 to $300 \mathrm{~ms}$, the ANOVA showed a main effect of regularity in the bilateral

superior temporal gyrus (STG) and an interaction between musical experience and

regularity in the left STG. These areas showed stronger activation in response to omission in regular than in irregular sequences (Fig. 4B). From 100 to $200 \mathrm{~ms}$ and 200 to $300 \mathrm{~ms}$, an interaction between musical experience and regularity was found in the left STG (Fig. 4C). No main effects or interactions were detected from 300 to $400 \mathrm{~ms}$. 
311 The peak coordinates of the activated areas are listed in Table 1.

317 ROI activity was analyzed using separate two-way ANOVAs with the factors musical sequences. For the regular sequences, the ANOVA showed main effects of musical for the irregular sequences showed no significant differences. 
329 three-way ANOVA with the factors musical experience, regularity, and position of

330 omission. No significant difference in activation was observed for each $100 \mathrm{~ms}$ time

331 window from 100 to $400 \mathrm{~ms}$ after tone onset, indicating that musical training did not

332 yield greater cortical activation by the pure tones employed in the present experiment.

333

334

335

\section{Discussion}

336 Both behavioral and neurophysiological differences were observed in the processing of

337 omissions between regular and irregular sequences. Better detection performance and

338 larger ERFs were associated with omissions in regular sequences than in irregular

339 sequences. Source-level analysis showed that omissions in the regular sequences

340 elicited stronger activation in the bilateral HG/STG than did those in the irregular

341 sequences. Based on predictive coding theory, these results can be interpreted to

342 indicate that activity in the auditory cortex is related to the matching between an internal

343 predictive model and an actual stimulus input.

346 We expected that the repetition of $\mathrm{C}, \mathrm{E}$, and $\mathrm{G}$ tones in a fixed order (CEGCEG...) 
347 would elicit perceptual grouping of the pitch pattern (CEG). The participants' faster

348 detection performance for the regular sequence agrees with the results of previous

349 studies, which have shown that perceptual grouping improves the detection and

350 recognition of target stimuli (Idson and Massaro, 1976; Jones et al., 1982; Mondor and

351 Terrio, 1998; Royer and Garner, 1970). In addition, the participants reported

352 recognizing the regular sequence as a repetition of a CEG pattern. Therefore, we believe

353 that the fixed order presentation of the $\mathrm{C}, \mathrm{E}$, and $\mathrm{G}$ tones in the regular sequence elicited

354 perceptual grouping of the CEG pattern.

From a predictive coding perspective, our results can be interpreted as follows:

the perceptual grouping of the CEG pattern strengthened top-down modulation from

higher-level brain areas and allowed stronger predictions to be created about incoming

tones than for the irregular sequences. Disagreement between this prediction and the input caused a larger prediction error in the regular sequence, resulting in a larger OR.

This was reflected by the significant activation difference around the bilateral HG/STG in comparing the prediction with the stimulus input. The meaning of the activation in previous study has examined the involvement of this region in perceptual grouping. 
365 Future research may clarify the importance of the region for this process.

applied to results of experiments in which the participants were instructed to ignore the

et al., 2009). For example, Bendixen et al. (2009) used repetition of a pair of tones with tones. This predictability-dependent difference was observed within $100 \mathrm{~ms}$ after omission onset, suggesting the occurrence of stimulus-driven prediction. Our findings can also be interpreted in light of this theory: in the present case, the prediction was created by modulation from a higher cortical level, such as attentional modulation. The latency difference of the OR between Bendixen et al (2009) and the present study may reflect this difference in type of prediction. This explanation would be in line with that of Bregman (1990), who suggested two mechanisms for perceptual grouping, a rapid stimulus-driven mechanism and a slower mechanism based on higher cognitive functions such as voluntary attention and/or experience-based knowledge. In light of the predictive coding theory and Bregman's theory, the results of Bendixen et al (2009) may reflect stimulus-driven prediction and pre-attentive perceptual grouping, while our findings may reflect top-down prediction and attentive perceptual grouping. 


\subsection{Impact of musical experience on perceptual grouping}

385 For omission in the regular sequence, musicians showed stronger activation in the left

386 STG than did nonmusicians, suggesting a stronger contribution of the left auditory

387 cortex in perceptual grouping. This result is in line with those of previous studies, which

388 have shown that the left hemisphere contributes to musical processing in musicians

389 during behavioral tasks (Bever and Chiarello, 1974; Burton et al., 1989; Messerli et al.,

390 1995) and neuroimaging studies (Boh et al., 2011; Evers et al., 1999; Hirshkowitz et al.,

391 1978; Matsui et al., 2013; Ono et al., 2011; Vuust et al., 2005). Left-hemisphere

392 dominance in analytical listening and right-hemisphere dominance in holistic listening

393 have been proposed, as has the idea that musicians' left hemisphere contribution to

394 auditory processing reflects an analytical listening strategy that differs from the holistic

395 listening strategy of nonmusicians (Bever and Chiarello, 1974; Burton et al., 1989;

396 Johnson, 1977; Messerli et al., 1995; Morais et al., 1982; Peretz and Morais, 1983).

397 Musical training generally includes the structural analysis of musical phrases as well as

398 practice with musical instruments. This training may induce analytical listening of tone musicians than in nonmusicians. 
be involved. Because the rate of correct detection was over $95 \%$, the task may have case, it would have been difficult to find significant differences between the groups. A more complicated task requiring more cognitive resources may have led to a significant

407 difference between musicians and nonmusicians at the behavioral level. Additionally, 408 the difference of listening strategy between musicians and nonmusicians may not have 409 led to differing performance in the detection task. Finally, the fact that all musicians 410 were amateurs may have contributed to the incongruity of the behavioral and MEG data.

411 Although we did not question the participants regarding the length of their musical 412 training per day or week, they were not trained as intensively as professional musicians.

413 This degree of musical training may have been insufficient to result in a behavioral 414 difference. the tone-evoked response. This result is not surprising as the effects of musical

417 experience appear in a use-dependent manner (Lütkenhöner et al., 2006; Pantev et al., 418 2001, 1998). For example, Pantev et al. (1998) found an increase in the N1 response to 
419 piano tones in musicians that did not occur for pure tones. While piano tones are often

420 encountered in musical training, pure tones are rarely experienced. Thus, after long-term

421 musical training, more neurons may be involved in processing musical stimuli, while no

422 change may occur for the brain processing of pure tones.

423

424

425 5. Conclusions

426 In summary, the perceptual grouping of pitch pattern in a tone sequence affected the

427 processing of omissions in the sequence, both behaviorally and neurophysiologically.

428 Our findings are in general agreement with those of earlier work suggesting the

429 predictive nature of the auditory system. In addition, our results suggest that perceptual

430 grouping elicited higher predictability for tones in a regular sequence, allowing for the

431 faster detection of omissions, and also engaged the bilateral HG/STG in comparing the

prediction and stimulus. Musical experience also influenced the neural processing of omissions, possibly reflecting a difference in listening strategy acquired through long-term musical training. 
438 The authors thank Ms. Yu Fukuda for her assistance with the programming of the

439 experiment and MEG measurement. This work was supported by the Japan Society for

440 the Promotion of Science (JSPS) KAKENHI Grant-in-Aid for Young Scientists (B)

441 (24730618) to K.O. The authors have no conflicts of interest to declare.

442

443

444

445

446

447

448

449

450

451

452

453 


\section{References}

456

457

458

459

460

461

462

463

464

465

466

467

468

469

470

471

472

473

Alain, C., Richer, F., Achim, A., Saint Hilaire, J.M., 1989. Human intracerebral potentials associated with target, novel, and omitted auditory stimuli. Brain Topogr. 1, 237-245.

Bendixen, A., SanMiguel, I., Schröger, E., 2012. Early electrophysiological indicators for predictive processing in audition: a review. Int. J. Psychophysiol. 83, 120-131.

Bendixen, A., Schröger, E., Winkler, I., 2009. I heard that coming: event-related potential evidence for stimulus-driven prediction in the auditory system. J. Neurosci. 29, 8447-51.

Bever, T.G., Chiarello, R.J., 1974. Cerebral dominance in musicians and nonmusicians. Science 21, 94-97.

Bhatara, A., Boll-Avetisyan, N., Unger, A., Nazzi, T., Höhle, B., 2013. Native language affects rhythmic grouping of speech. J. Acoust. Soc. Am. 134, 3828-3843.

Boh, B., Herholz, S., Lappe, C., Pantev, C., 2011. Processing of complex auditory patterns in musicians and nonmusicians. PLoS One 6, e21458.

Bregman, A., 1990. Auditory scene analysis. The perceptual organization of sound. MIT Press, Cambridge MA.

Burton, A., Morton, N., Abbess, S., 1989. Mode of processing and hemisphere differences in the judgement of musical stimuli. Br. J. Psychol. 80, 169-180.

Deutsch, D., 2012. The psychology of music, 3rd ed. Academic Press.

Dewar, K.M., Cuddy, L.L., Mewhort, D.J., 1977. Recognition memory for single tones with and without context. J. Exp. Psychol. Hum. Learn. 3, 60-67.

Evers, S., Dannert, J., Rödding, D., Rötter, G., Ringelstein, E.B., 1999. The cerebral haemodynamics of music perception. A transcranial Doppler sonography study. Brain 122, 75-85. 
480

481

482

483

484

485

486

487

488

489

490

491

492

493

494

495

496

497

498

499

500

501

502

503

504

505

506

507

Friston, K., 2005. A theory of cortical responses. Philos. Trans. R. Soc. Lond. B. Biol. Sci. 360, 815-836.

Friston, K., Harrison, L., Daunizeau, J., Kiebel, S., Phillips, C., Trujillo-Barreto, N., Henson, R., Flandin, G., Mattout, J., 2008. Multiple sparse priors for the M/EEG inverse problem. Neuroimage 39, 1104-20.

Friston, K., Kiebel, S., 2009a. Cortical circuits for perceptual inference. Neural Networks 22, 1093-1104.

Friston, K., Kiebel, S., 2009b. Predictive coding under the free-energy principle. Philos. Trans. R. Soc. Lond. B. Biol. Sci. 364, 1211-21.

Gobet, F., Simon, H.A., 1996. Recall of rapidly presented random chess positions is a function of skill. Psychon. Bull. Rev. 3, 159-163.

Hirshkowitz, M., Earle, J., Paley, B., 1978. EEG alpha asymmetry in musicians and non-musicians: A study of hemispheric specialization. Neuropsychologia 16, $125-128$.

Hughes, H.C., Darcey, T.M., Barkan, H.I., Williamson, P.D., Roberts, D.W., Aslin, C.H., 2001. Responses of human auditory association cortex to the omission of an expected acoustic event. Neuroimage 13, 1073-1089.

Idson, W.L., Massaro, D.W., 1976. Cross-octave masking of single tones and musical sequences: The effects of structure on auditory recognition. Percept. Psychophys. $19,155-175$.

Iversen, J.R., Patel, A.D., Ohgushi, K., 2008. Perception of rhythmic grouping depends on auditory experience. J. Acoust. Soc. Am. 124, 2263-2271.

Johnson, P.R., 1977. Dichotically-stimulated ear differences in musicians and nonmusicians. Cortex 13, 385-389.

Jones, M.R., Boltz, M., Kidd, G., 1982. Controlled attending as a function of melodic and temporal context. Percept. Psychophys. 32, 211-218.

Joutsiniemi, S.-L., Hari, R., 1989. Omissions of auditory stimuli may activate frontal cortex. Eur. J. Neurosci. 1, 524-528. 
508

509

510

511

512

513

514

515

516

517

518

519

520

521

522

523

524

525

526

527

528

529

530

531

532

533

534

535

Koffka, K., 1935. Principles of Gestalt psychology. Hartcourt Brace, New York, NY.

Laird, A.R., Robinson, J.L., McMillan, K.M., Tordesillas-Gutiérrez, D., Moran, S.T., Gonzales, S.M., Ray, K.L., Franklin, C., Glahn, D.C., Fox, P.T., Lancaster, J.L., 2010. Comparison of the disparity between Talairach and MNI coordinates in functional neuroimaging data: validation of the Lancaster transform. Neuroimage $51,677-683$.

Lancaster, J.L., Tordesillas-Gutiérrez, D., Martinez, M., Salinas, F., Evans, A., Zilles, K., Mazziotta, J.C., Fox, P.T., 2007. Bias between MNI and Talairach coordinates analyzed using the ICBM-152 brain template. Hum. Brain Mapp. 28, 1194-1205.

Lütkenhöner, B., Seither-Preisler, A., Seither, S., 2006. Piano tones evoke stronger magnetic fields than pure tones or noise, both in musicians and non-musicians. Neuroimage 30, 927-937.

Matsui, T., Tanaka, S., Kazai, K., Tsuzaki, M., Katayose, H., 2013. Activation of the left superior temporal gyrus of musicians by music-derived sounds. Neuroreport 24, $41-45$.

Mattout, J., Phillips, C., Penny, W.D., Rugg, M.D., Friston, K.J., 2006. MEG source localization under multiple constraints: an extended Bayesian framework. Neuroimage 30, 753-767.

Messerli, P., Pegna, A., Sordet, N., 1995. Hemispheric dominance for melody recognition in musicians and non-musicians. Neuropsychologia 33, 395-405.

Mondor, T.A., Terrio, N.A., 1998. Mechanisms of perceptual organization and auditory selective attention: The role of pattern structure. J. Exp. Psychol. Hum. Percept. Perform. 24, 1628-1641.

Morais, J., Peretz, I., Gudanski, M., 1982. Ear asymmetry for chord recognition in musicians and nonmusicians. Neuropsychologia 20, 351-354.

Ono, K., Nakamura, A., Yoshiyama, K., Kinkori, T., Bundo, M., Kato, T., Ito, K., 2011. The effect of musical experience on hemispheric lateralization in musical feature processing. Neurosci. Lett. 496, 141-145. 
536

537

538

539

540

541

542

543

544

545

546

547

548

549

550

551

552

553

554

555

556

557

558

559

560

561

562

Pantev, C., Oostenveld, R., Engelien, A., Ross, B., Roberts, L.E., Hoke, M., 1998. Increased auditory cortical representation in musicians. Nature 392, 811-814.

Pantev, C., Roberts, L.E., Schulz, M., Engelien, A., Ross, B., 2001. Timbre-specific enhancement of auditory cortical representations in musicians. Neuroreport 12, $169-174$.

Pantev, C., Ross, B., Fujioka, T., Trainor, L.J., Schult, M., Schulz, M., 2003. Music and learning-induced cortical plasticity. Ann. N. Y. Acad. Sci. 999, 438-450.

Penney, T.B., 2004. Electrophysiological correlates of interval timing in the Stop-Reaction-Time task. Brain Res. Cogn. Brain Res. 21, 234-249.

Peretz, I., Morais, J., 1983. Task determinants of ear differences in melody processing. Brain Cogn. 2, 313-330.

Raij, T., McEvoy, L., Mäkelä, J.P., Hari, R., 1997. Human auditory cortex is activated by omissions of auditory stimuli. Brain Res. 745, 134-143.

Royer, F., Garner, W., 1970. Perceptual organization of nine-element auditory temporal patterns. Percept. Psychophys. 7, 115-120.

Saariluoma, P., 1989. Chess player's recall of auditorily presented chess positions. Eur. jounal Cogn. Psychol. 1, 309-320.

Shaffer, J., 1986. Modified sequentially rejective multiple test procedures. J. Am. Stat. Assoc. 81, 826-831.

Simon, H.A., Chase, W.G., 1973. Skill in Chess. Am. Sci. 61, 394-403.

Snyder, J.S., Large, E.W., 2005. Gamma-band activity reflects the metric structure of rhythmic tone sequences. Brain Res. Cogn. Brain Res. 24, 117-126.

Tarkka, I.M., Stokic, D.S., 1998. Source localization of P300 from oddball, single stimulus, and omitted-stimulus paradigms. Brain Topogr. 11, 141-151.

Todorovic, A., van Ede, F., Maris, E., de Lange, F.P., 2011. Prior expectation mediates neural adaptation to repeated sounds in the auditory cortex: an MEG study. J. Neurosci. 31, 9118-9123. 
563

564

565

566

567

568

569

570

571

572

573

574

575

576

577

578

579

580

581

582

583

584

585

586

Vuust, P., Pallesen, K.J., Bailey, C., van Zuijen, T.L., Gjedde, A., Roepstorff, A., Østergaard, L., 2005. To musicians, the message is in the meter pre-attentive neuronal responses to incongruent rhythm are left-lateralized in musicians. Neuroimage 24, 560-564.

Wacongne, C., Labyt, E., van Wassenhove, V., Bekinschtein, T., Naccache, L., Dehaene, S., 2011. Evidence for a hierarchy of predictions and prediction errors in human cortex. Proc. Natl. Acad. Sci. U. S. A. 108, 20754-20759.

Winkler, I., 2007. Interpreting the Mismatch Negativity. J. Psychophysiol. 21, 147-163.

Winkler, I., Czigler, I., 2012. Evidence from auditory and visual event-related potential (ERP) studies of deviance detection (MMN and vMMN) linking predictive coding theories and perceptual object representations. Int. J. Psychophysiol. 83, 132-143.

Winkler, I., Denham, S.L., Nelken, I., 2009. Modeling the auditory scene: predictive regularity representations and perceptual objects. Trends Cogn. Sci. 13, 532-540.

Yabe, H., Tervaniemi, M., Reinikainen, K., Näätänen, R., 1997. Temporal window of integration revealed by MMN to sound omission. Neuroreport 8, 1971-1974.

Yabe, H., Winkler, I., Czigler, I., Koyama, S., Kakigi, R., Sutoh, T., Hiruma, T., Kaneko, S., 2001. Organizing sound sequences in the human brain: the interplay of auditory streaming and temporal integration. Brain Res. 897, 222-227. (1) 


\section{Legends}

$589 \quad$ Fig. 1

590 Sequence of stimuli and behavioral performance in the detection task. A. Sequence of

591 stimuli used in the experiment. In the regular sequence, $\mathrm{C}, \mathrm{E}$, and $\mathrm{G}$ tones were

592 presented as a repetition of a CEG pattern, whereas in the irregular sequence the tones

593 were presented pseudo-randomly. B. Reaction time in the detection task. Error bars

594 display the standard error of the mean (SEM). $\mathrm{M}=$ musicians; $\mathrm{NM}=$ nonmusicians. $\mathbf{C}$.

595 Examples of the magnetoencephalography (MEG) waveform evoked by the omission of

596 the $\mathrm{C}$ tone in the irregular sequence for one musician. Waveforms from 20 gradiometers

597 that covered the temporal lobe in each hemisphere were superimposed.

598

$599 \quad$ Fig. 2

600 Time course of the root mean square (RMS) amplitude of the omission-related response

601 (OR). A. RMS amplitude of the OR in musicians. B. RMS amplitude of the

602 omission-related response in nonmusicians.

603

$604 \quad$ Fig. 3

605 RMS amplitude of the brain response evoked by the omission between 100 and $400 \mathrm{~ms}$ 
606 after omission onset. The asterisks represent $p<0.001$.

607

608 Fig. 4

609 Reconstructed maps showing significantly activated brain areas and the results of the

610 three-way analysis of variance (ANOVA) with the factors musical experience, regularity,

611 and position of omission. A. Visualization of the brain areas significantly activated

612 between 100 and $400 \mathrm{~ms}$ after sound omission in the regular and irregular sequences, as

613 determined by one-sample $t$ tests (uncorrected $p<0.005$ ). B. Brain areas showing a

614 main effect of regularity in the three-way ANOVA for the time windows from 100 to

$615200 \mathrm{~ms}$ and from 200 to $300 \mathrm{~ms}$ (uncorrected $p<0.001$ ). C. Brain areas showing an

616 interaction between musical experience and regularity in the three-way ANOVA for the

617 time windows from 100 to $200 \mathrm{~ms}$ and from 200 to $300 \mathrm{~ms}$ (uncorrected $p<0.001$ ). $\mathrm{L}=$

618 left; $\mathrm{R}=$ right.

619

$620 \quad$ Fig. 5

621 Mean amplitude of the region of interest (ROI) activity. The ROI was located in the left

622 superior temporal gyrus (STG), which was defined by the brain area showing the

623 interaction between musical experience and regularity in the three-way ANOVA for the 
$33 / 33$

624 time windows from 100 to $200 \mathrm{~ms}$ and from 200 to $300 \mathrm{~ms}$ (Fig. 4C). $\mathrm{M}=$ musicians;

$625 \mathrm{NM}=$ nonmusicians. A.U. $=$ arbitrary unit. 
A Regular sequence
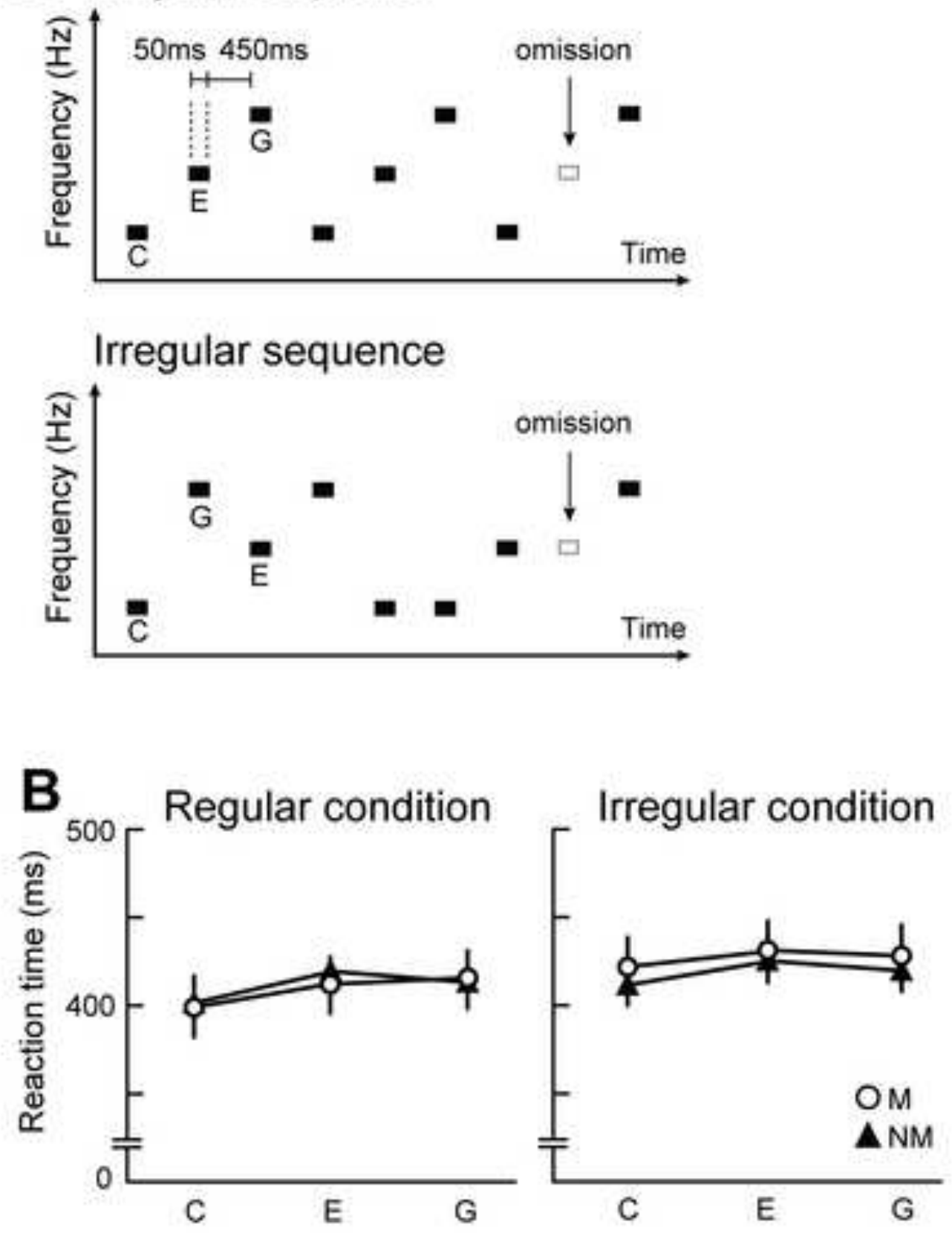

C Omission-related response (omission of $\mathrm{C}$ tone)

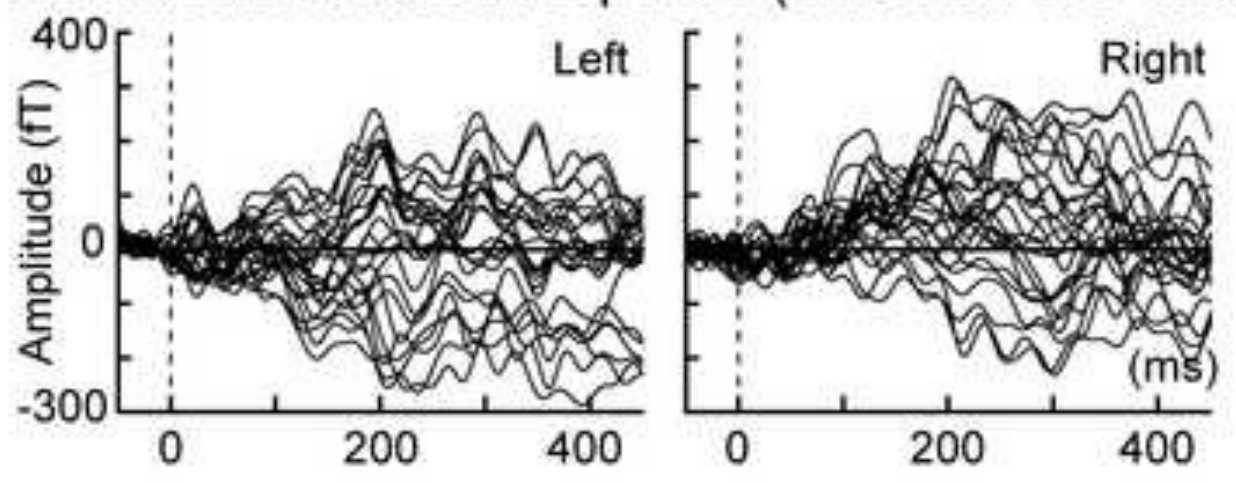


A Musicians - Regular … Irregular
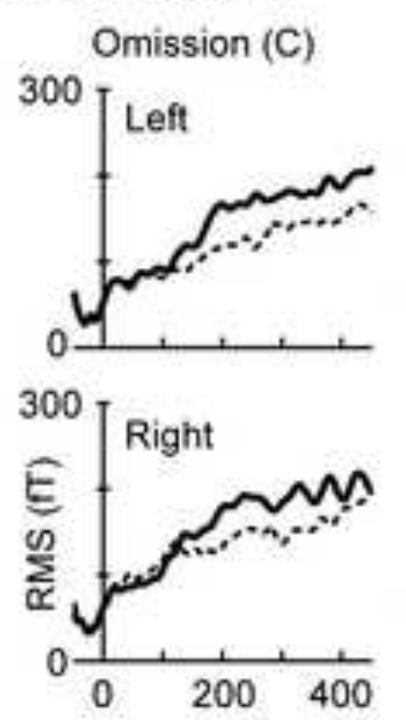

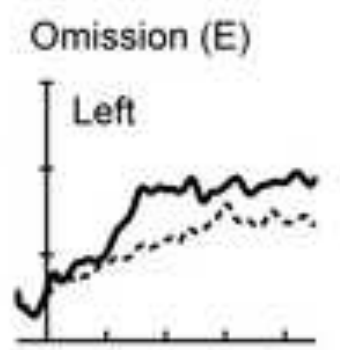

Omission (G)
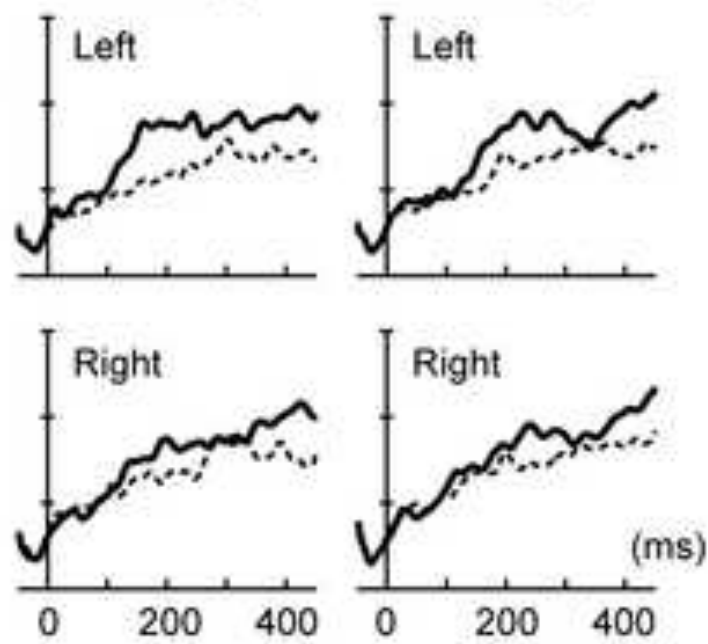

B Nonmusicians - Regular … Irregular

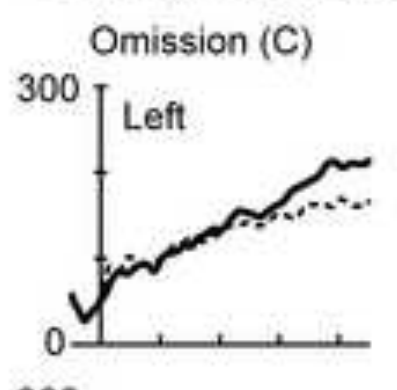

Omission (E)

Omission (G)
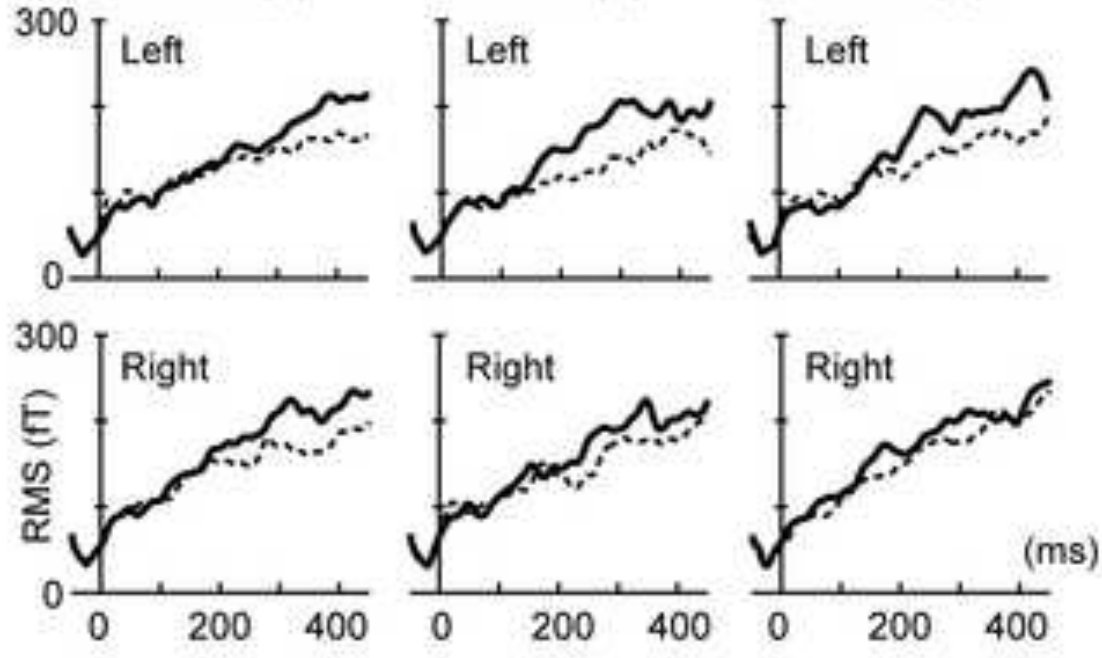
$\square$ Omission (C) $\square$ Omission (E) DOmission (G)

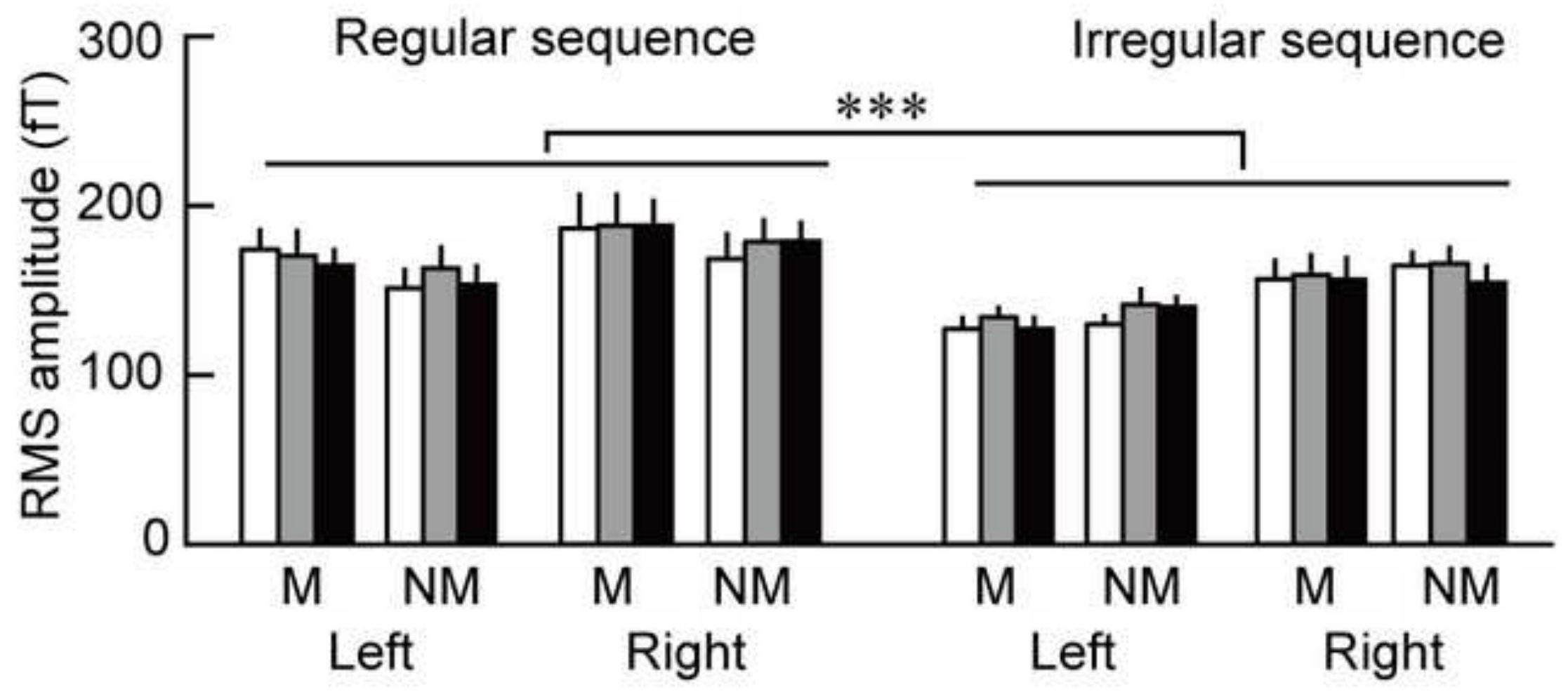




\section{A Musicians}
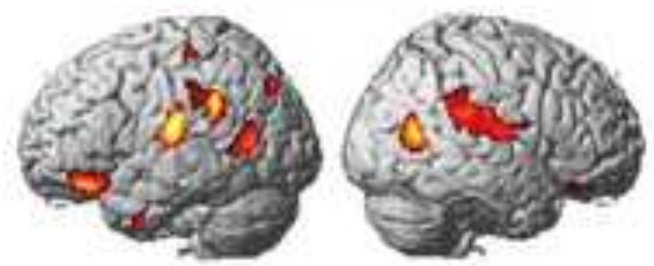

Regular
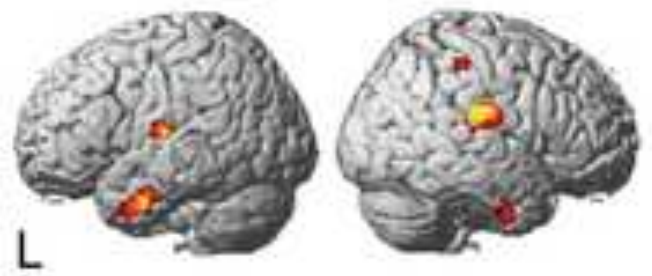

\section{Nonmusicians}

Irregular
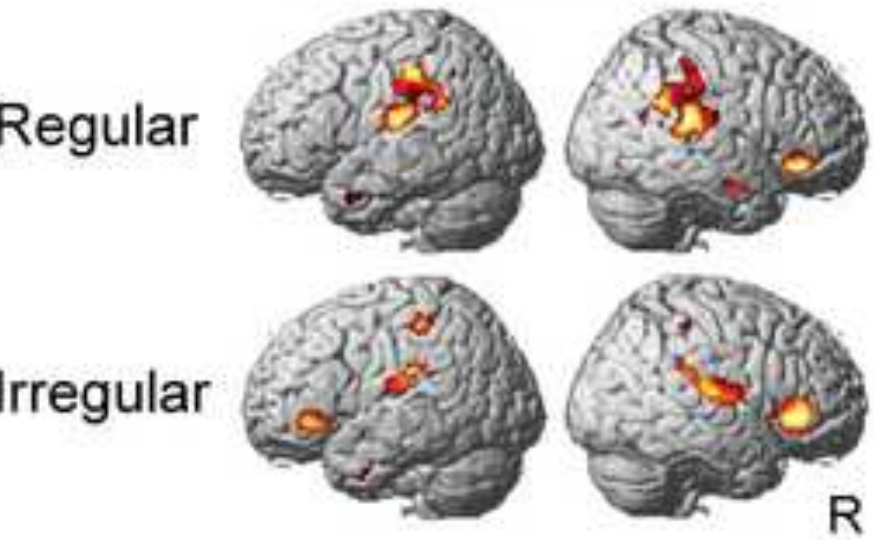

B Main effect of regularity (Regular > Irregular)

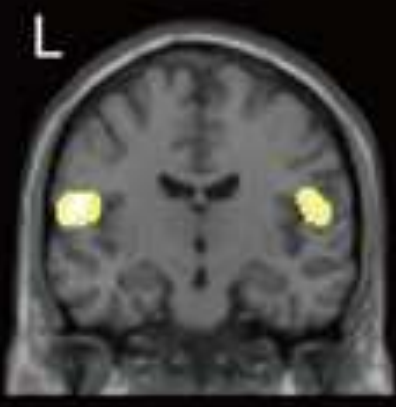

$100-200 \mathrm{~ms}$

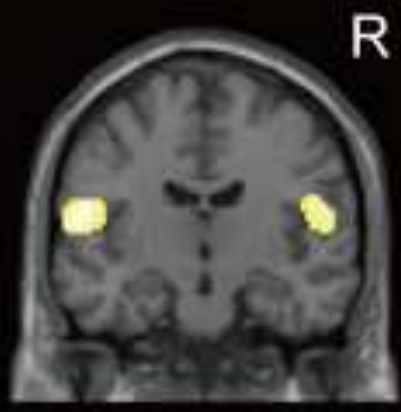

$200-300 \mathrm{~ms}$ $z$ value

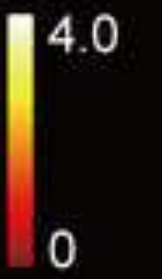

C Interaction between experience and regularity

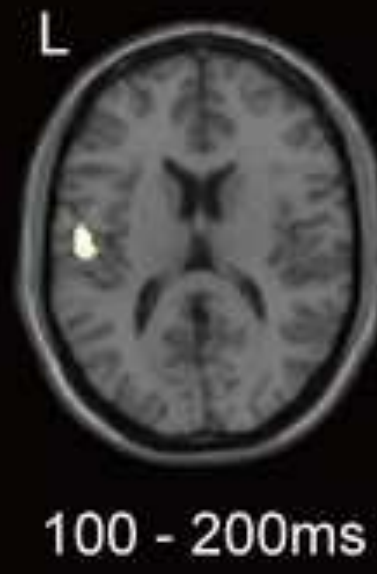

$100-200 \mathrm{~ms}$

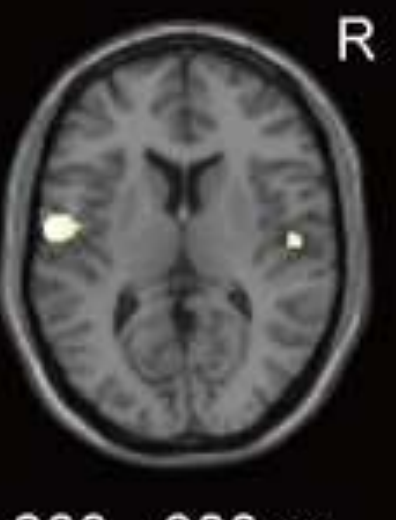

$200-300 \mathrm{~ms}$ $z$ value

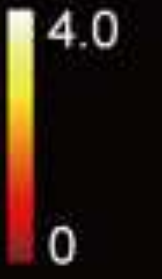




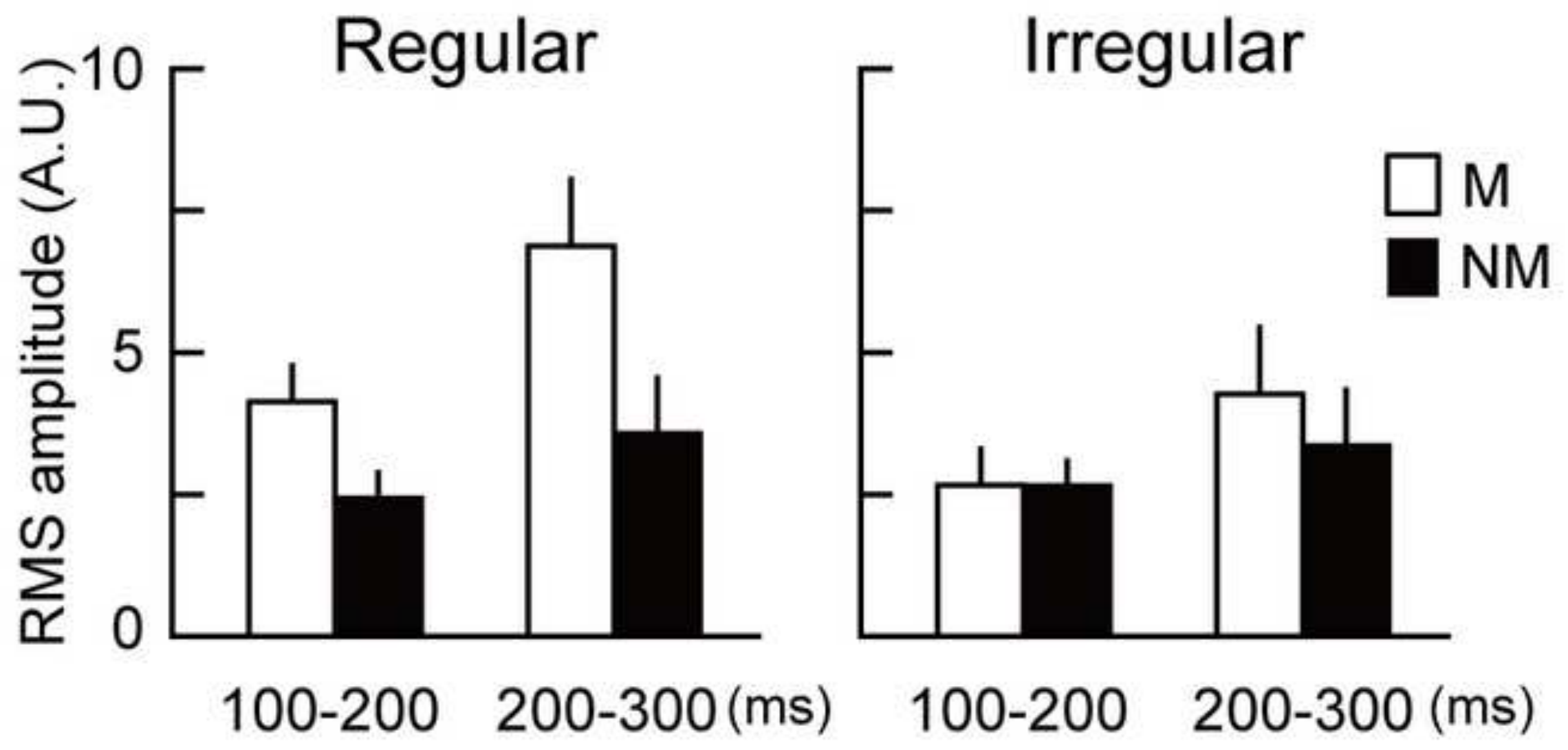




\section{Table 1}

Peak coordinates of significantly activated areas for each 100-ms time window in the three-way ANOVA with factors musical experience, regularity, and position of the omission (uncorrected $p<0.001$ ).

Brain area (Brodmann's Area $[\mathrm{BA}]) \quad$ Peak coordinates $(x, y, z)$

Number of voxels $z$ value

\section{0-200 ms}

Main effect of regularity (Regular > Irregular)

$\begin{array}{lccccc}\text { Left Heschl's gyrus [BA41] } & -51 & -16 & 13 & 426 & 3.87 \\ \text { Right postcentral gyrus [BA43] } & 48 & -12 & 18 & 57 & 3.30\end{array}$

Interaction of musical experience and regularity

([Regular-Irregular] in musicians > [Regular - Irregular] in nonmusicians $)$

$\begin{array}{llllll}\text { Left Heschl's gyrus [BA41] } & -51 & -16 & 13 & 142 & 3.51\end{array}$

\section{0-300 ms}

Main effect of regularity (Regular > Irregular)

$\begin{array}{llllll}\text { left superior temporal gyrus [BA22] } & -53 & -11 & 8 & 561 & 4.06 \\ \text { right superior temporal gyrus [BA22] } & 50 & -12 & 9 & 328 & 3.66\end{array}$

Interaction of musical experience and regularity 
([Regular - Irregular] in musicians > [Regular - Irregular] in nonmusicians )

left superior temporal gyrus [BA22]

$-55$

$-7$

6

209

3.43 